\title{
Optical properties of magnetoexcitons in double quantum dots
}

\author{
Piotr Schillak ${ }^{\mathrm{a}}$ and Gerard Czajkowski \\ UTP University of Science and Technology, Institute of Mathematics and Physics, Al. Prof. S. Kaliskiego 7, \\ 85-789 Bydgoszcz, Poland
}

Received 13 February 2015 / Received in final form 14 May 2015

Published online 7 October 2015

(c) The Author(s) 2015. This article is published with open access at Springerlink.com

\begin{abstract}
Here we investigate the influence of a constant magnetic field on the energy levels and optical properties of excitons in a double quantum disk (quantum molecule). Taking into account the cylindrical symmetry of the double disk we calculate the wave functions and excitonic energies when the external constant magnetic field is applied along the symmetry axis. Having the eigenfunctions and eigenvalues and using the long-wave approximation we can compute all the optical functions. The double quantum dot is considered as one system rather than two interacting dots separated by narrow barrier. The screened Coulomb interaction between an electron and a hole is assumed. Since in the given structure the separation of the relative- and center-of-mass motion of the electron and the hole is not possible, we use an approach where the six-dimensional eigenvalue problem is transformed into the equivalent eigenvalue problem given by the system of the coupled two-dimensional second order differential equations. The so obtained differential equations are solved numerically. As an example, we give detailed results for a InP/InGaP double quantum dot. Satisfactory agreement with the available experimental data is obtained.
\end{abstract}

\section{Introduction}

The development of the optoelectronic devices gains the interest in the electronic and optical properties of various semiconductor nanostructures including quantum wires, quantum rods, quantum dots and double quantum dots. The influence of excitonic effects on the optical functions (oscillator strengths, absorption coefficient, dielectric susceptibility, etc.) is very important from both experimental and theoretical points of view [1-8]. The presence of external static electric and/or magnetic fields significantly modifies the electronic structure and optical spectra of nanostructures and a number of investigations has been carried out to understand and describe the problem. The influence of the magnetic field on the optical spectra of magnetoexcitons in quantum disks was analyzed theoretically and investigated experimentally in a series of papers. The (far from completeness) list of references can be found, for example, in reference [9]. Recently, the technological progress gives the possibility of creating more complicated structures than single quantum dots, quantum disks, etc. The simplest example of such a structure is a pair of quantum dots, also called a double quantum dot, see, for example [10]. More complicated structures as, for example, triple and quadruple quantum dots, or triple quantum rings, can also be fabricated and investigated (for example [11-13]). All the problems, related to the electronic and optical properties, can also be investigated in the case of the mentioned multiple quantum

\footnotetext{
a e-mail: piotr.schillak@utp.edu.pl
}

nanostructures. Here we consider the optical properties of a double quantum dot, using the approach described in reference [9].

The proposed theoretical approach enables to calculate the magnetic field dependent excitonic energy spectrum and wave functions. Having wave functions we are able to determine the magnetic field dependent excitonic susceptibility and the complex dielectric function.

The paper is organized as follows. In Section 2 we present the theoretical model and briefly describe the transformation of the six-dimensional eigenvalue problem to the equivalent system of coupled two-dimensional second order differential equations. Numerical examples for a chosen double quantum dots are presented in Section 3. We present our conclusions in Section 4.

\section{The model}

We consider the double quantum dot (quantum molecule) exhibiting cylindrical symmetry, which is exposed to the external static magnetic field applied along the symmetry axis (Fig. 1). The interacting electron-hole pair inside the quantum molecule forms the magnetoexciton characterized by its own energy spectrum and the corresponding wave functions, which are dependent on six coordinates. The effective masses of both charged particles are in general anisotropic and usually different for the disks and for the barrier material. The steplike confinement potential at the disks boundaries has a finite value, different for an 


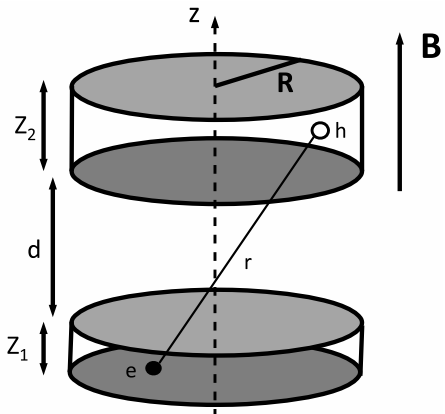

Fig. 1. Magnetoexciton in a double quantum dot.

electron and a hole. The analytical calculations for a quantum molecule are very similar to those, which were performed for the nanostructures exhibiting cylindrical symmetry and presented in reference [9]. Here we repeat the basic equations, briefly describe the method of solution and give some remarks on numerical computations.

The Hamiltonian describing the system is as follows:

$$
\hat{H}=\hat{H}_{e}+\hat{H}_{h}+V_{e h},
$$

where $V_{e h}=-e^{2} /(4 \pi \varepsilon|\mathbf{r}|)$ is the screened Coulomb interaction potential. Making use of cylindrical coordinates $\mathbf{r}_{j}=\left(\rho_{j}, \phi_{j}, z_{j}\right)$, we write the one-particle Hamiltonians in the form

$$
\begin{aligned}
\hat{H}_{j}= & \hat{H}_{j \|}+\hat{H}_{j z}+V_{j, \text { conf }}, \\
\hat{H}_{j \|}= & \frac{1}{2}\left(\hat{\mathbf{p}}_{j}-q_{j} \hat{\mathbf{A}}_{j}\right) \underline{\underline{m}}_{j \|}^{-1}\left(\hat{\mathbf{p}}_{j}-q_{j} \hat{\mathbf{A}}_{j}\right) \\
= & -\frac{\hbar^{2}}{2}\left(\frac{1}{\rho_{j}} \frac{\partial}{\partial \rho_{j}} \frac{\rho_{j}}{m_{\| j}} \frac{\partial}{\partial \rho_{j}}+\frac{1}{\rho_{j}^{2} m_{\| j}} \frac{\partial^{2}}{\partial \phi_{j}^{2}}\right. \\
& \left.+\frac{\mathrm{i} \hbar q_{j} B}{2 m_{\| j}} \frac{\partial}{\partial \phi_{j}}+\frac{q_{j}^{2} B^{2}}{8 m_{\| j}} \rho_{j}^{2}\right)
\end{aligned}
$$

$\left(j=e, h ; q_{j}=\mp e\right)$. The $z$-axis is parallel to the symmetry axis and to the external magnetic field. The mass tensors $\underline{\underline{m}}_{j}=\operatorname{diag}\left(m_{\| j}, m_{\| j}, m_{z j}\right)$ elements have different values inside and outside the nanostructure. We use the steplike confinement potentials $V_{j, \text { conf }}\left(\rho_{j}, z_{j}\right)=0$ inside the quantum dots and $V_{j, \text { conf }}=V_{0, j}$ outside the quantum dots. The dots, separated by the barrier of the thickness $d$, have the same radius $R$, the heights are $z_{1}$ and $z_{2}$ as it is shown in Figure 1 . The vector potential $\mathbf{A}_{j}=\frac{1}{2} \mathbf{B} \times \mathbf{r}_{j}$ is taken in the symmetrical gauge and the magnetic field $\mathbf{B}=(0,0, B)$. We introduce dimensionless energies scaled in exciton Rydberg energy $R^{*}$ and spatial variables $\varrho=\rho / a_{B}^{*}$ scaled in the excitonic Bohr radius $a_{B}^{*}$ using the following formulae:

$$
\begin{aligned}
& a_{B}^{*}=\frac{4 \pi \varepsilon_{0} \sqrt{\varepsilon_{\|} \varepsilon_{z}}}{m_{0} \mu_{\|} e^{2}}=\frac{\sqrt{\varepsilon_{\|} \varepsilon_{z}}}{\mu_{\|}} a_{0}, \\
& R^{*}=\frac{\mu_{\|}}{\varepsilon_{\|} \varepsilon_{z}} \frac{m_{0} e^{4}}{2\left(4 \pi \varepsilon_{0}\right)^{2} \hbar^{2}}=\frac{\mu_{\|}}{\varepsilon_{\|} \varepsilon_{z}} R_{\infty},
\end{aligned}
$$

where $\varepsilon_{0}, \epsilon_{z}, \varepsilon_{\|}$are the vacuum dielectric constant, relative dielectric constant in the $z$-direction, relative dielec- tric constant in the direction perpendicular to the $z$-axis, respectively; $m_{0}$ is the free electron mass,

$$
\mu_{\|}=m_{\| e} m_{\| h} /\left(m_{\| e}+m_{\| h}\right)
$$

is the reduced mass expressed in $m_{0}$ units; $a_{0}$ and $R_{\infty}$ are the hydrogen atom Bohr radius and Rydberg energy, respectively. Using the scaled variables we can write the two-particle Schrödinger equation in the form

$$
\left(-\frac{\mu_{\|}}{m_{z e}} \frac{\partial^{2}}{\partial z_{e}^{2}}-\frac{\mu_{\|}}{m_{z h}} \frac{\partial^{2}}{\partial z_{h}^{2}}+\hat{H}_{\| e}+\hat{H}_{\| h}-\frac{2}{r}+v_{c}\right) \Psi=\epsilon \Psi,
$$

where $\hat{H}_{\| j}$ are the Hamiltonians defined in equation (3), $\epsilon$ is the scaled energy, $v_{c}=\left(V_{e, \text { conf }}+V_{h, \text { conf }}\right) / R^{*}$, and $r$ is the relative e-h distance:

$$
r=\sqrt{\varrho_{e}^{2}+\varrho_{h}^{2}-2 \varrho_{e} \varrho_{h} \cos \left(\phi_{e}-\phi_{h}\right)+\left(z_{e}-z_{h}\right)^{2}} .
$$

It can be shown (see, for example, Ref. [9]) that the two dimensional eigenvalue problem $\hat{H}_{\|} \psi=\epsilon \psi$ of the charged particle in a circular well surrounded by the steplike potential can be solved analytically. So, we can expand the six dimensional wave function $\Psi$ in equation (6) in terms of two dimensional eigenfunctions for an electron and a hole as follows:

$$
\Psi^{(L)}=\sum_{\mathbf{n}} f_{\mathbf{n}}^{(L)}\left(z_{e}, z_{h}\right) \psi_{n_{1} m_{1}}^{(e)}\left(\varrho_{e}, \phi_{e}\right) \psi_{n_{2} m_{2}}^{(h)}\left(\varrho_{h}, \phi_{h}\right) .
$$

The index $\mathbf{n}$ is the abbreviation for four indices, i.e. $\mathbf{n}=\left\{n_{1}, m_{1}, n_{2}, m_{2}\right\}$, where $n_{1}=0,1,2, \ldots, m_{1}=$ $0, \pm 1, \pm 2, \ldots$ and $n_{2}=0,1,2, \ldots, m_{2}=0, \pm 1, \pm 2, \ldots$ are the quantum numbers describing the electron and the hole states in a circular well, respectively. The total angular momentum is conserved because of the axial symmetry of the problem, so $L=m_{1}+m_{2}$ is a good quantum number and the sum contains only the terms with fixed $L$, as marked in the superscripts.

We insert the expansion (8) into equation (6) and obtain the equations for the functions $f_{\mathbf{n}}^{(L)}\left(z_{e}, z_{h}\right)$ :

$$
\begin{aligned}
\left(-\mu_{\|} \frac{\partial}{\partial z_{e}}\right. & \frac{1}{m_{z e}} \frac{\partial}{\partial z_{e}}-\mu_{\|} \frac{\partial}{\partial z_{h}} \frac{1}{m_{z h}} \frac{\partial}{\partial z_{h}}+\epsilon_{n_{1} m_{1}}^{(e)} \\
& \left.+\epsilon_{n_{2} m_{2}}^{(h)}+v_{\perp c}\right) f_{\mathbf{n}}^{(L)}+\sum_{\mathbf{n}^{\prime}} V_{\mathbf{n}}^{\mathbf{n}^{\prime}} f_{\mathbf{n}^{\prime}}^{(L)}=\epsilon f_{\mathbf{n}}^{(L)}
\end{aligned}
$$

where $\epsilon_{n_{1} m_{1}}^{(e)}, \epsilon_{n_{2} m_{2}}^{(h)}$ are the energies of an electron and a hole in a two dimensional well, respectively. The matrix elements $V_{\mathbf{n}}^{\mathbf{n}^{\prime}}\left(z_{e}, z_{h}\right)$ of Coulomb interaction energy are fourfold integrals which can be reduced to triple integrals by changing variables and performing one integration analytically [9]. The shape of the diagonal matrix element $V_{1}^{1}(z)$ is displayed in Figure 2. From Figure 2 one can see that the coupling between subsequent equations became weaker with increasing value of the upper index. It means that we can solve numerically only several equations to get satisfactory accuracy. 


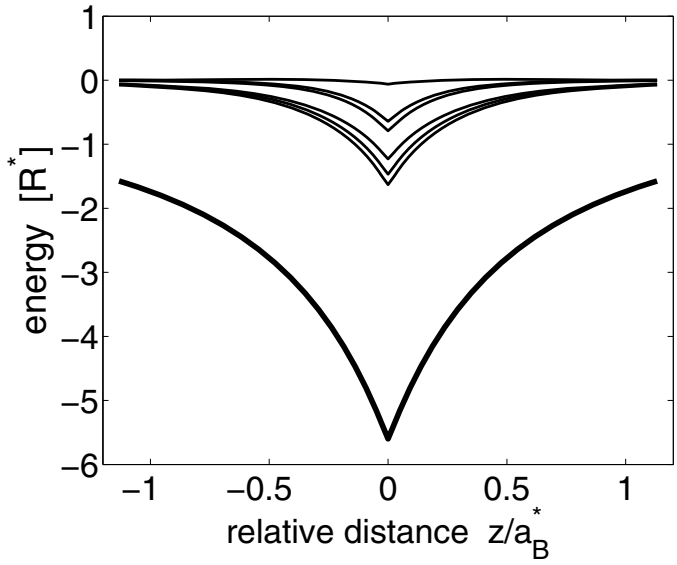

Fig. 2. Coulomb interaction matrix elements $V_{1}^{j}$, the lowest curve corresponds to diagonal element $V_{1}^{1}(z)$, the next are $V_{1}^{2}, V_{1}^{3}, \ldots$

The system of coupled partial differential equations (9) is the eigenvalue problem for the set of functions $f_{\mathbf{n}}^{(L)}\left(z_{e}, z_{h}\right)$ and for the total energy (eigenvalue) $\epsilon$ of the electron-hole pair inside the nanostructure. The method of solution of equation (9) has been described in reference [9] (see also, for example, Ref. [14]). The method uses the discretization in space and a conversion of differential equations into a set of linear algebraic equations. The discretization was performed in the volume of a sufficiently large cylinder containing both quantum dots. The cylinder is coaxial with the $z$-axis of the quantum molecule.

\section{Results and discussion}

The above described calculation scheme was applied to the problem of the InAs/GaAs two quantum disks with the radii $R$, thicknesses $z_{1}$ and $z_{2}$, interdot distance $d$; exposed to uniform static magnetic field parallel to the $z$-axis. The basic band parameters (effective masses, band mismatch, dielectric constant) for the electron and the heavy hole were taken to be the same as in the paper by Thu and Voskoboynikov [15]: inside the dot: $m_{e}=0.044 m_{0}, m_{h h}=$ $0.254 m_{0}, V_{e, \text { conf }}=474 \mathrm{meV}, V_{h, \text { conf }}=203 \mathrm{meV}$, outside the dot: $m_{e}=0.067 m_{0}, m_{h h}=0.350 m_{0}, m_{0}$ being the free electron mass. We take the averaged dielectric constant $\varepsilon=14.0$. We obtained the eigenfunctions and energy states from numerical solution of equations (9). We studied the influence of the applied magnetic field strength and the in- $z$ direction confinement shape (the structure symmetric or asymmetric) on the energy levels and the shape of the wavefunctions for the ground and excited states. In Figure 3c we display the energy levels as functions of the applied magnetic field for the symmetric shape molecule. We checked that every presented level is doubly degenerated. The degeneracy appears because the upper and lower quantum dots (see Fig. 1) are identical, so we have the same probability to find the particle in the upper or lower disk as can be seen in Figures 3a and 3b. Additionally the Coulomb interaction between the dots is weak because of the relatively thick $(d=5.0 \mathrm{~nm})$ separating

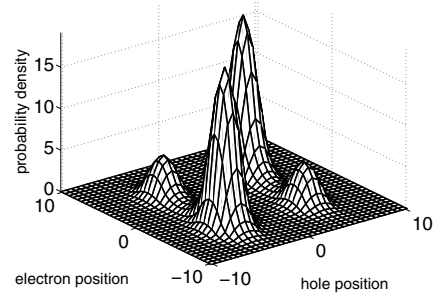

(a)

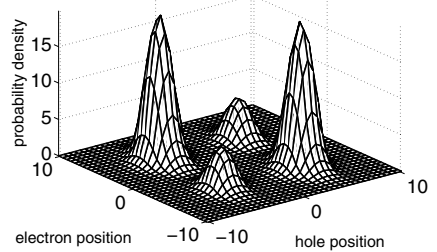

(b)

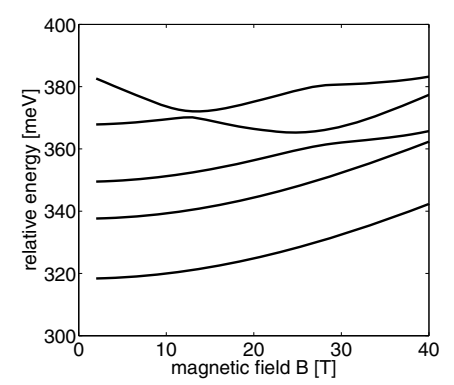

(c)

Fig. 3. Probability density projection on the $\left(z_{e}, z_{h}\right)$ plane for magnetic field $B=2 \mathrm{~T}$. The molecule is symmetrical: $z_{1}=$ $z_{2}=3.0 \mathrm{~nm}, d=5.0 \mathrm{~nm}$ : (a) ground state, (b) first excited state. (c) Relative positions of (almost doubly degenerated) energy levels as functions of the applied magnetic field.

barrier. The degeneracy of the levels is removed when the separating barrier is thinner as it can be seen in Figure 4 where $d=3.0 \mathrm{~nm}$. In this case the particles can tunnel through the barrier between the lower and upper dot, additionally the Coulomb interaction is stronger.

The same as in Figure 3 is displayed in Figure 5 for an asymmetric shape of the double dot (the disks of different heights). We observe differences as compared to the symmetric shape, both in the wave functions and in energy diagram. This is not visible in the scale of the presented figures but the curves representing energy versus magnetic field do not cross each other. The anticrossing of energy states is due to Coulomb interaction.

Having the energies and wave functions, we can use the Fermi Golden Rule or, alternatively, the real density matrix approach (see, for example, Refs. [16-18]), to obtain the optical functions, including magnetoabsorption and the magnetic field dependent dielectric tensor for the considered nanostructure. The results for the imaginary parts of the magnetosusceptibility are shown in Figure 6.

Experimental investigations of magnetooptical properties of nanostructures have shown that the main effect on the ground state is the blue-shift of the energy, roughly proportional to $B^{2}$, with a slope which is specific for the given nanostructure and depends on the configuration (Faraday or Voigt). Such effect was observed for quantum wells (see, for example, Ref. [19]), quantum dots [20] and quantum disks [21]. It was also observed for a system of $\mathrm{InP} / \mathrm{InGaP}$ two vertically stacked dots $[22,23]$, which corresponds to the situation discussed above. The detailed analysis performed in a series of papers (see, for example, 


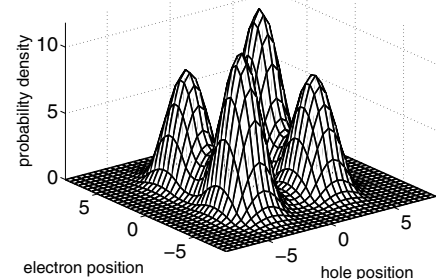

(a)

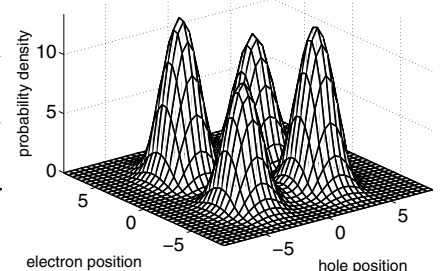

(b)

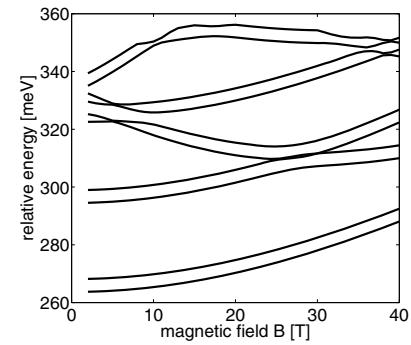

(c)
Fig. 4. Probability density projection on the $\left(z_{e}, z_{h}\right)$ plane for magnetic field $B=2 \mathrm{~T}$. The molecule is symmetrical: $z_{1}=z_{2}=3.0 \mathrm{~nm}, d=3.0 \mathrm{~nm}$ : (a) ground state, (b) excited state. (c) Relative positions of energy levels as functions of the applied magnetic field.

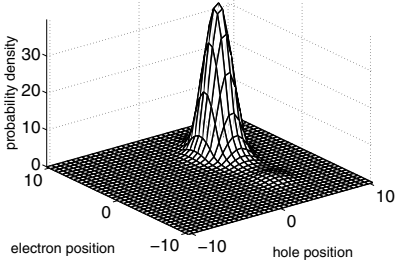

(a)

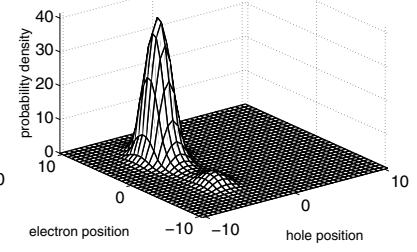

(b)

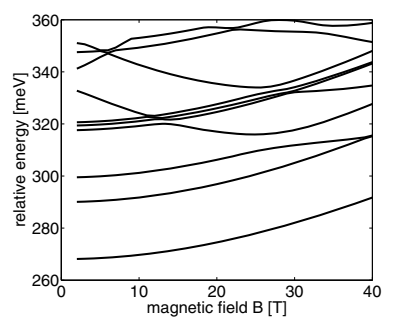

(c)

Fig. 5. Probability density projection on the $\left(z_{e}, z_{h}\right)$ plane for magnetic field $B=2 \mathrm{~T}$. The molecule is asymmetrical: $z_{1}=$ $3.0 \mathrm{~nm}, z_{2}=3.2 \mathrm{~nm}, d=5.0 \mathrm{~nm}$ : (a) ground state, (b) excited state. (c) Relative positions of energy levels as functions of the applied magnetic field.

Refs. $[10,24,25])$ shows that the ground state is determined by the light hole located outside the dots, so we have a type-II configuration for the exciton. Our model was primarily developed for type-I exciton, but it also can be used to describe type-II exciton when we assume that the dots are the barriers for the light hole. We have performed numerical computations using the material parameters listed

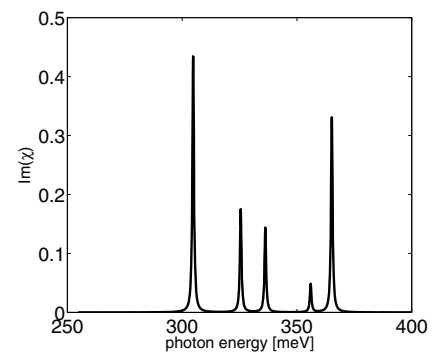

(a)

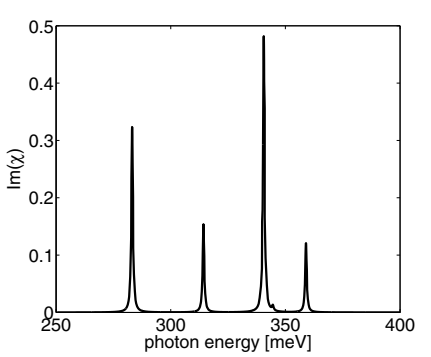

(b)

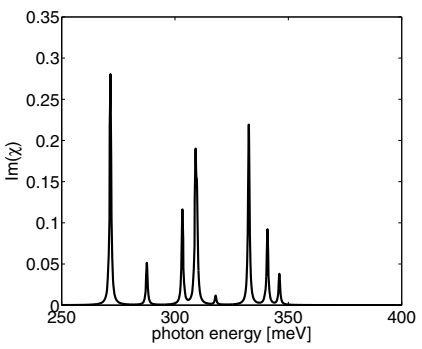

(c)

Fig. 6. Imaginary part of magnetosusceptibility corresponding to Figures $3-5$ for $B=5 \mathrm{~T}$.

in Table I of reference [24], for the conduction-band effective masses we used $0.0795 m_{0}$ for the electron inside the dot and $0.092 m_{0}$ outside the dot [10]. We assumed rectangular barriers and their heights, calculated within CM (continuum mechanical) model are taken from reference [25]. Our numerical calculations showed that we obtain the same results for light and heavy holes as presented in reference [24] for two vertically coupled dots. The slightly better agreement with experimental data can be obtained if we assume that the disk radius is equal to $7 \mathrm{~nm}$; the result is presented in Figure 7. As it is displayed in Figure 7, using our computational scheme, we are able to fit the experimental results from references $[22,23]$ for a quite large interval of the applied magnetic field strength (up to $35 \mathrm{~T}$ ). For large values of the magnetic field we observe a deviation from the parabolic behaviour. The deviation from parabolic shape of the diamagnetic shift for high magnetic field can not be explained within our model. One of the reasons is that we apply the single band effective mass approximation. The approach proposed by Mlinar et al. [10] is more adequate to describe the nanostructures when type-I and type-II exciton configuration should be taken into account.

\section{Concluding remarks}

We have applied a calculational scheme, developed for the case of cylindrically symmetric nanostructures, to a double quantum dot. The double dot was not considered as a two-particles structure, but it was modelled by an appropriate shape of the confinement potential. The method allows to compute the eigenfunctions, the energy levels and thus the optical properties. In addition, the effects of an applied static magnetic field have been 


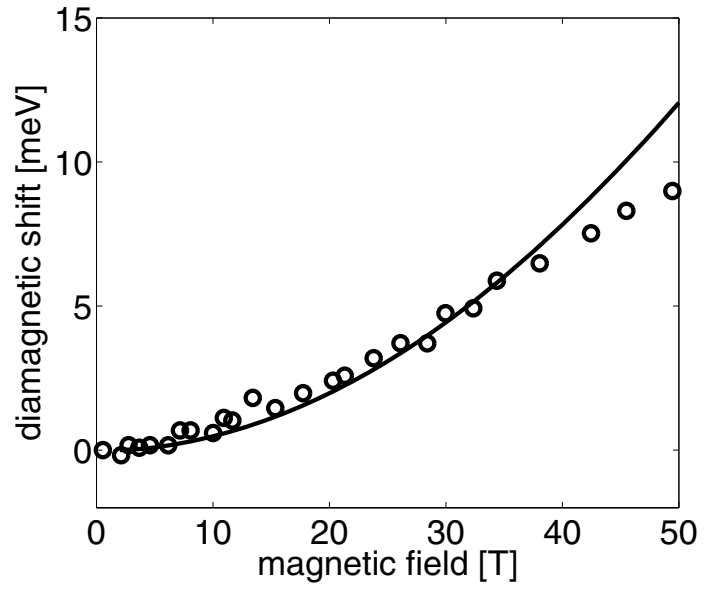

Fig. 7. Comparison of the calculated (full line) diamagnetic shift with experimental results $[22,23]$ (circles) for $\mathrm{InP} / \mathrm{InGaP}$ double quantum dot. The dots are identical with radius $R=7 \mathrm{~nm}$ and thickness $d=2 \mathrm{~nm}$, the interdot distance is equal to $4 \mathrm{~nm}$.

calculated. The advantage of the method is that not only the ground energy state, but also higher energy states, can be obtained. Modelling triple and quadruple quantum dots by an appropriate confinement potential, we can use the same method to obtain the optical properties in the excitonic energy region. The numerical calculations have been performed for a structure consisting of two InAs/GaAs quantum disks with the radii $R$, thicknesses $z_{1}$ and $z_{2}$, interdot distance $d$; exposed to uniform static magnetic field parallel to the $z$-axis. The impact of the type of the structure (symmetric or asymmetric) and of the interdot distance has been discussed. As it has been shown in previous papers, the method is exact and the convergence can be controlled. The results are in a qualitative agreement with those obtained by a different approach as, for example, in references $[24,26]$.

\section{Author contribution statement}

Both authors designed the research and contributed to the development of the model and data interpretation. P. Schillak designed the algorithms and performed the numerical calculations, and was the leading writer of the manuscript.

Numerical computations were carried out at the Academic Computer Center in Gdańsk, Poland.

\section{References}

1. L. Woggon, Optical Properties of Semiconductor Quantum Dots (Springer-Verlag, Berlin, 1997)

2. L. Jacak, P. Hawrylak, A. Wojs, Quantum Dots (SpringerVerlag, Berlin, 1998)
3. D. Bimberg, M. Grundmann, N.N. Ledentsov, Quantum Dot Heterostructures (Wiley, New York, 1998)

4. T. Chakraborty, Quantum Dots (Elsevier, Amsterdam, 1999)

5. V.M. Ustinov, A.E. Zhukov, A.Yu. Egorov, N.A. Maleev, Quantum Dot Lasers (Oxford University Press, Oxford, 2003)

6. E.L. Wolf, Nanophysics and Nanotechnology, An Introduction to Modern Concepts in Nanoscience (Wiley, Weinheim, 2004)

7. O. Manasreh, Semiconductor Heterojunctions and Nanostructures (McGraw-Hill, New York, 2005)

8. P. Harrison, Quantum Wells, Wires and Dots (McGrawHill, New York, 2005)

9. P. Schillak, Eur. Phys. J. B 84, 17 (2011)

10. V. Mlinar, M. Tadić, F.M. Peeters, Phys. Rev. B 73, $235336(2006)$

11. M. Tadić, F.M. Peeters, Phys. Rev. B 70, 195302 (2004)

12. M. Tadić, F.M. Peeters, Phys. Rev. B 79, 153305 (2009)

13. B. Szafran, E. Barczyk, F.M. Peeters, S. Bednarek, Phys. Rev. B 77, 115441 (2008)

14. S. Glutsch, Excitons in Low-Dimensional Semiconductors, Theory, Numerical Methods, Applications (Springer, Berlin, 2004)

15. L. M. Thu, O. Voskoboynikov, Phys. Rev. B 80, 155442 (2009)

16. A. Stahl, I. Balslev, Electrodynamics of the Semiconductor Band Edge (Springer-Verlag, Berlin, Heidelberg, New York, 1987)

17. G. Czajkowski, F. Bassani, L. Silvestri, Rivista del Nuovo Cimento 26, 1 (2003)

18. P. Schillak, G. Czajkowski, J. Phys.: Conf. Ser. 210, 012016 (2010)

19. O. Jaschinski, M. Vergöhl, J. Schoeness, A. Schlachetzki, Phys. Rev. B 57, 13086 (1998)

20. R. Cingolani, R. Rinaldi, H. Lipsanen, M. Sopanen, R. Virkkala, K. Maijala, J. Tulkki, J. Ahopelto, K. Uchida, N. Miura, Y. Arakawa, Phys. Rev. Lett. 83, 4832 (1999)

21. P.D. Wang, J.L. Merz, S. Fafard, R. Leon, D. Leonard, G. Medeiros-Ribeiro, M. Oestreich, P.M. Petroff, K. Uchida, N. Miura, H. Akiyama, H. Sakaki, Phys. Rev. B 53, 16458 (1996)

22. M. Hayne, R. Provoost, M.K. Zundel, Y.M. Manz, K. Eberl, V.V. Moshchalkov, Phys. Rev. B 62, 10324 (2000)

23. M. Hayne, R. Provoost, M.K. Zundel, Y. Manz, K. Eberl, V.V. Moshchalkov, Physica E 6, 436 (2000)

24. K.L. Janssens, B. Partoens, F.M. Peeters, Phys. Rev. B 69, $235320(2004)$

25. M. Tadić, F.M. Peeters, K.L. Janssens, Phys. Rev. B 65, $165333(2002)$

26. L.M. Thu, O. Voskoboynikov, Comput. Mater. Sci. 49, S281 (2010)

Open Access This is an open access article distributed under the terms of the Creative Commons Attribution License (http://creativecommons.org/licenses/by/4.0), which permits unrestricted use, distribution, and reproduction in any medium, provided the original work is properly cited. 